\title{
Erratum to: Perennial wheat lines have highly admixed population structure and elevated rates of outcrossing
}

\author{
J. Piaskowski $\cdot$ Kevin Murphy $\cdot$ Theodore Kisha $\cdot$ Stephen Jones
}

Published online: 17 August 2017

(C) Springer Science+Business Media B.V. 2017

\section{Erratum to: Euphytica (2017) 213:171 DOI 10.1007/s10681-017-1961-x}

Due to an unfortunate turn of events this article was published with omissions in the Abstract text. Therefore the following Abstract should be regarded as the final version by the reader.

Abstract Perennial wheat has been proposed to alleviate long standing issues with soil erosion in annual cropping

The online version of the original article can be found under doi:10.1007/s10681-017-1961-x.

J. Piaskowski ( $)$

Department of Horticulture, Washington State University, Pullman, WA 99164-6414, USA

e-mail: jpiaskowski@wsu.edu

K. Murphy $\cdot$ S. Jones

Department of Crop \& Soil Sciences, Washington State

University, Pullman, WA 99164-6420, USA

e-mail: kmurphy2@wsu.edu

S. Jones

e-mail: joness@wsu.edu

T. Kisha

Western Plant Introduction Station, USDA-ARS,

Pullman, WA 99164-6402, USA

e-mail: theodore.kisha@ars.usda.gov

S. Jones

11768 Westar Lane, Unit E, Box 5, Burlington,

WA 98233, USA systems, while supporting rural communities and providing grain farmers with a marketable climate-resilient crop. The Washington State University perennial wheat breeding program has created several hundred interspecific progeny by crossing several different cultivars of winter wheat (Triticum aestivum L.) with Thinopyrum species and $\times$ Agrotriticum spp. Prior to this study, the chromosome composition of these wheat-wheatgrass derivatives was not characterized, limiting their utility as stable breeding germplasm. We determined the mitotic chromosome number and species origin of chromosomes for eight breeding lines, and estimated their relatedness and population structure using AFLPs. Additionally, self-pollination and outcrossing rates were estimated for these breeding lines to gain an understanding of perennial wheat's reproductive strategy. We intercrossed the lines with each other to produce 20 families and then measured the level of chromosome pairing during meiosis I in the F1 progeny. The lines contained between 44 and 64 chromosomes, of which eight to 16 were from Th. ponticum. Our analysis of molecular diversity indicated greater genetic diversity within, rather than across, breeding lines (88 and 12\%, respectively). The outcrossing rate was estimated at $16 \%$. Understanding chromosome number and origin is necessary for developing a population of breeding lines that can be used as parents. Our results suggest that the perennial wheat breeding lines act as a single diverse population that can be improved using breeding strategies for inbred and outcrossing crops. 